\title{
Domain modeling based on Engineering Standards
}

\author{
Carlos Toro ${ }^{1}$, Manuel Graña ${ }^{3}$, Jorge Posada ${ }^{1}$, Javier Vaquero ${ }^{1}$, Cesar Sanín ${ }^{2}$ \\ Edward Szczerbicki ${ }^{2}$ \\ ${ }^{1}$ VICOMTech Research Centre, Spain \\ ${ }^{2}$ Faculty of Engineering and Built Environment, University of Newcastle, Australia \\ ${ }^{3}$ University of the Basque Country - Facultad de Informática de San Sebastián, Spain
}

\begin{abstract}
In this paper we present a new methodology for the Domain modeling based on Engineering Standards. We discuss some benefits of standards as guidelines for a Knowledge Based Domain modeling and some potential challenges along with possible approaches to overcome them. The benefits of use of Standards as models for Domain ontologies have been shown valid in related works and as prove of concept we present a case study where our methodology was applied successfully.
\end{abstract}

Keywords. Domain Modeling, Knowledge Based Systems, ontologies

\section{Introduction}

Webster Dictionary defines a Domain as "a sphere of knowledge, influence or activity". In Computer Science, we consider a Domain as a sphere of Knowledge identified by a name, where the contained information is a collection of concepts, intermediations and facts about entities [8]. In other words, a Domain describes the elements and characteristics belonging to a Knowledge Base. A Domain ontology (or Domain-specific ontology) models the Knowledge in a specific Domain, representing particular meanings for the contained terms. For example, in the Plant Design Domain, the concept elbow is a specific type of bend pipe used to change the direction of the fluid. When considering similar Domains, the degree of specialization and conceptualization even with the same concept can slightly vary (e.g. a piping engineer will consider a wider definition of an elbow when compared to a structural engineer). The Domain modeling does not stop only in the concept definition. Any concept in a Domain also needs the properties characterization e.g. for an elbow in the Plant Design Domain, distinctiveness like the radius, the curvature, etc. In this paper we will present a methodology that can be used to aid in the Domain modeling of a Knowledge Base using Engineering Standards. The paper is constructed as follows: In section 2 we will present a state of the art on the different topics relevant to our work. In section 3, we present our methodology for the Domain modeling based on Engineering Standards. In section 4 we present a case study where we successfully 
applied our methodology, and lastly in section 5 we present some conclusions and future work.

\section{State of the art}

In this section we introduce some background concepts relevant to this paper. Knowledge is considered an invaluable resource of great benefit for most purposes in life. For this reason, mankind has always attempted to make it part of their assets. Knowledge itself seems to be an attribute of human beings; it may be defined [7] as: (i) the expertise and skills acquired by a person through experience or education via a theoretical or practical understanding of a subject, (ii) what is known in a particular field or in total related to facts and information or (iii) the awareness or familiarity gained by experience of a fact or situation. Knowledge Engineering (KE) is an engineering discipline that involves integrating Knowledge into computer systems in order to solve complex problems, normally requiring a high level of human expertise [2]. Following that line of thought, Knowledge Bases can be modeled and used by computer systems in order to enhance their capacities. One of the most used techniques for Knowledge modeling is ontologies.

\subsection{Knowledge modeling using ontologies}

We base our approach in the accepted and widespread definition of ontology given by Tom Gruber widespread accepted definition of what ontology is in the Computer Science Domain: an ontology is the explicit specification of a conceptualization; in other words is a description of the concepts and relationships in a Domain [3]. Some of the reasons to use ontologies in Knowledge modeling are: (i) To separate Domain Knowledge from the operational Knowledge, (ii) to analyze Domain Knowledge, (iii) to share common understanding of the structure of information among people or software agents, $(i v)$ to enable reuse of Domain Knowledge, and $(v)$ to make Domain assumptions explicit. To our knowledge, there are few reported cases where Standards are used along with semantic technologies, being the most notorious the case of CIDOC-CRM [7], which is a formal ontology intended to facilitate the integration, mediation and interchange of heterogeneous cultural heritage information whose primary role is to enable information exchange and integration between heterogeneous sources of cultural heritage information. The usual approach for Domain modeling starts with human experts who use their own knowledge about the special needs of an industry in order to model the subjects and their relations using editors and even plain paper. In some companies the Domain expert is known as the "Knowledge engineer" and his purpose is to conceptualize the bushiness now-how and processes. 


\subsection{Engineering Standards}

According to BSI [1], a standard is an agreed, repeatable way of doing something. It is a published document that contains a technical specification or other precise criteria designed to be used consistently as a rule, guideline, or definition. Standards help to make life simpler and to increase the reliability and the effectiveness of many goods and services we use. Standards are created by bringing together the experience and expertise of all interested parties such as the producers, sellers, buyers, users and regulators of a particular material, product, process or service. Standards are designed for voluntary use and do not impose any regulations. However, laws and regulations may refer to certain standards and make compliance with them compulsory. Any standard is a collective work. Committees of manufacturers, users, research organizations, government departments and consumers work together to draw up standards that evolve to meet the demands of society and technology. Typically Standards are categorized into different levels that roughly correspond to their spatial influence. A comprehensive list of standards organizations can be found in [5]. Between the most relevant standardization organizations we can mention: DIN, which is the German Standards organization, CAM-I, which is the international association for Manufacturing Standards, BSI, the British Standards organization (the oldest organization of this type in the world) and ISO, which is the International Standards Organization.

\section{A methodology for the use of Engineering Standards as models for Domain}

In this section, we introduce our methodology for the Domain modeling based on Engineering Standards.

\subsection{Reasons to use Standards as base for Domain}

The ability to demonstrate compliance with widely recognized and respected standards is an effective mean of differentiation in a competitive marketplace. In addition, manufacturing products or supplying services to appropriate standards maximizes their compatibility with those manufactured or offered by others, thereby increasing potential sales and widespread acceptance. As consumers become increasingly informed about their choices, compliance to recognized standards becomes pivotal. We argue that the use of Engineering Standards as models for the Domains provides the following benefits:

- Consensus: There is a consensus about the terminology, organization and logic of the Domain.

- $\quad$ Formats support: Many Engineering applications support Engineering Standars as input/output formats (e.g. CAD software with STEP modules [7]. This fact 
helps in the categorization of elements and the mapping of such elements into the Knowledge Base.

- Avoidance of Semantic loss: The modeling of an Engineering Standard, usually considers not only the element in isolation, but also, the relation of such element with surrounding objects. The aforementioned fact is indeed a very valuable feature that helps in the conservation of the Semantics properties of such elements.

- Easiness of a new Domain modeling based on existing Standards: If there is no existing Engineering Standard for a given Domain, a standard complying with similar characteristics can be used. An example to this is the use of STEP application protocol 227 (Plant Design) ([7], [8]) for the case of Ship Design.

- Standards are revised on a regular basis: Their nature is eminently evolutionary due to the development of new technologies for fabrication or the typical evolution of engineering paradigms. When using Standard as base for Knowledge Base, there exists an intrinsic guarantee of using the most recent data models.

Our methodology is divided in a series of logical steps that must be performed to assure a correct modeling of the Domain (see Fig. 1).

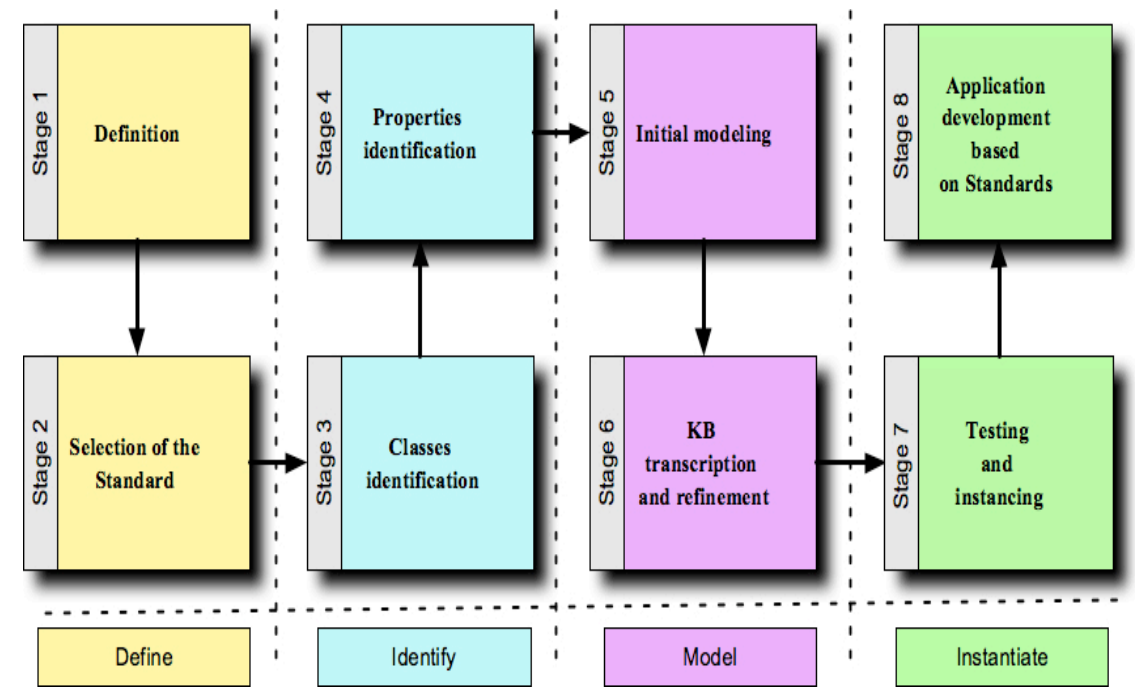

Fig. 1. Our methodology for Domain modeling based on Standards

As can be seen, we divided our methodology in 4 layers: DEFINE, IDENTIFY, MODEL and INSTANTIATE, encompassing eight stages as follows:

DEFINE:

- Stage 1- Definition: In this stage, an identification of the purpose and requirements of the domain is made. What is the purpose of the $\mathrm{KB}$, the information that will be stored and the needed level of detail of such information.

- Stage 2- Selection of the Standard: In this stage, there is a search of a standard that suits the defined needs. As a result of this selection, the chosen standard must be studied in detail, how is constructed, what can be done in order to extend it, etc 
IDENTIFY:

- Stage 3- Class identification: In this stage, an identification of the purpose and requirements of the domain is made. What is the purpose of the KB, the information that will be stored and the needed level of detail of such information.

- Stage 4- Property identification: At this stage, the characteristics that can be measured or determined by data types (string, Boolean value, integer, etc), are identified in each class, e.g. outside diameter, length, etc. Then we identify the characteristics that relate a class with other classes (relation types). In general data type characteristics are easily recognizable and obtained by simple interrogations (e.g. to a geometric model). Relation types are a little bit more difficult to find, because generally when talking about a geometric model whose characteristics will be obtained, those sets of elements are categorized as geometric primitives rather than functional objects. For the aforementioned case, solutions like the process of branding and matching MODEL: presented by Posada, could be used [4].

- Stage 5- Initial modeling: In this stage, a subset of the domain is chosen in order to verify the complexity of the overall modeling and the real capabilities of the KB. Since the elaboration of a $\mathrm{KB}$ is an iterative process by nature, this small test must answer initial modeling needs.

- Stage 6- KB transcription and refinement: Sometimes the initial modeling is enough for the $\mathrm{KB}$ to fulfill the design requirements in stage 1 , however is highly recommended to perform a verification of the transcription, by using for example the capabilities of a ontology reasoner to check the congruence of the $\mathrm{KB}$. Once the transcription is done, a refinement process takes place. In such stage any needed extension of the standard take place. Usually the transcription a refinement is performed using an editor.

\section{INSTANTIATE:}

- Stage 7- Testing and instancing: In this step the test of the instances and the creation of an automatic or semiautomatic instancing mechanism (if needed) is performed. As a final step, some individuals conforming to the specification of the classes can be manually modeled using again an editor. Such process can be automated if needed if any API tools are available and the elements that must conform can be interrogated e.g. modeling an industrial plant KB that has a $3 \mathrm{D}$ counterpart in $\mathrm{CAD}$, the CAD API can be used to interrogate the elements and the editor API to semi-automatically "fill" the individuals in the KB. This step does not strictly fall into the modeling process, but in order to really use the modeled KB is needed.

- Stage 8- Application development based on Standards: In this last step the Virtual Engineering Application using the Domain model is developed, this last stage comprises the actual usability of the Domain where the VEA advantages from Semantics via the enhancement obtained by having a better described and consensual Domain model.

\subsection{Potential challenges}

There are some potential challenges to take into account when modeling a Domain based on Engineering Standards, between others we can mention the following: $(i)$ The design of the Standard could be Functional-side balanced: In some cases, the Engineering Standards is functional oriented, a fact that leads to potential semantic loss, as the Standard doesn't include the needed parameters for a complete Domain modeling. The mentioned case is exemplified by the STEP element "Valve" [7]. In such element, the standard specifies only functional parameters (actuator_type, operation_mode, type), but lacks information about geometric parameters that are needed and also easy readable from a CAD model (diameter, length, etc.). For such case an extension of the class should be performed in order to obtain a complete Knowledge Base. When the extension of the Domain is needed, is advisable to double check if the parameter is a fundamental one. Sometimes, the Engineering Standards offers a way to obtain the needed parameter by interrogating neighbor elements (in the case of the Valve, the input and output pipes could be used for such purpose). If 
the parameter need is fundamental and the extension is unavoidable, it should be clearly specified as an "outside the standard feature" and it must follow the Engineering Standards architecture, e.g. it should be part of the correct element and moreover derived from the correct parent class. (ii) The Standard can disappear or being absorbed by other standard: Maybe for a lack of use or because of administrative reasons, some standards disappear (e.g. the case of CAM-I AIS); in such cases the use of a Knowledge Base based on such Engineering Standards could be continued, however it would be advisable to migrate the Knowledge Base to a new paradigm when available. In the case of absorption by other standard, the advisable is to review the model in order to check its robustness. (iii) The Standard falls short for the Domain needs: This indicates a possible immature Engineering Standards, or an inappropriate election by the Domain designer. For both cases, is advisable the reading and understanding of the Standard and an extensive review of the problem's characterization (requisites).

\section{Case Study}

In this section we will exemplify our proposed methodology for Domain modeling based on Engineering Standards.

Stage 1- Definition: Let us consider the problem of modeling an Industrial Plant and in particular for the example purposes the modeling of a Flange element. Stage 2 - Selection of the Standard: By performing an Internet search, we find that there is an ISO Standard that could be used for our needs; this case is ISO 10303 AP 227 (Industrial Plants) [8]. Stage 3 Classes identification: Searching in the standard we find that a description of a Flange element exists; such description is depicted in Fig. 2.

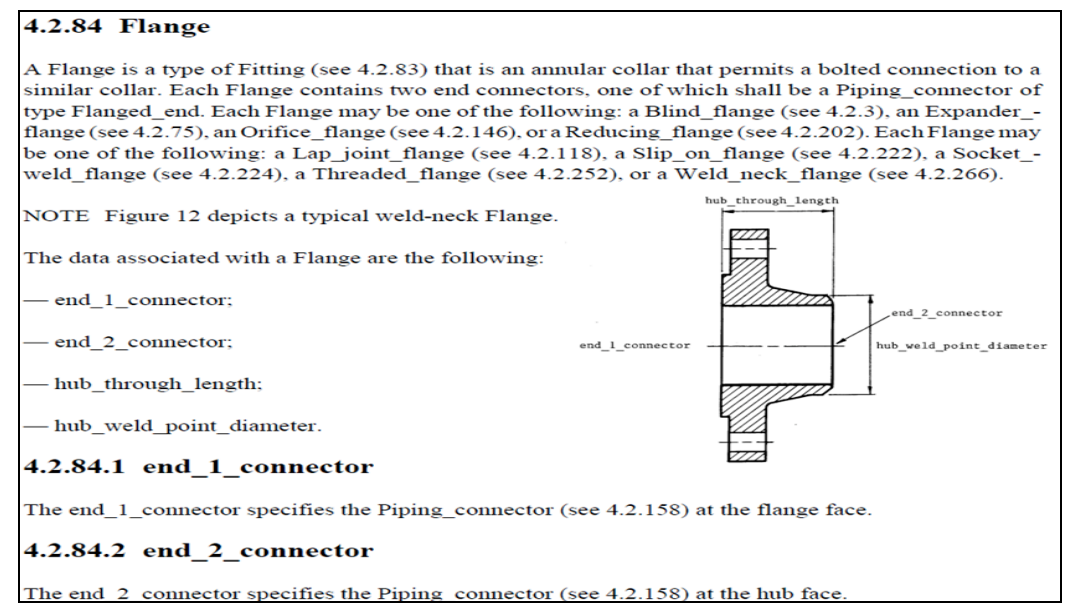

Fig. 2. STEP Excerpt for the case study

Stage 4 - Property identification: By looking at the properties in the Flange element, we create a classification as can be seen in Table 1 . 
Table. 1. Property classification

\begin{tabular}{|l|l|l|}
\hline Name & Property_type & Value \\
\hline hub_through_length & Data & Double \\
\hline hub_weld_point_diameter & Data & Double \\
\hline end_1_connector & Relational & Element \\
\hline end_2_connector & Relational & Element \\
\hline
\end{tabular}

Stage 5 - Initial modeling: We use the Protégé ontology editor to model the element as can be seen in Fig. 3,4, and 5.

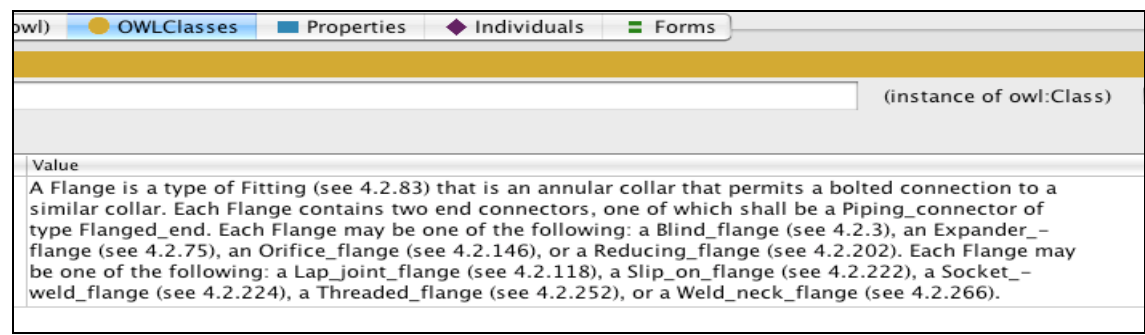

Fig. 3. Modeling of the Flange class

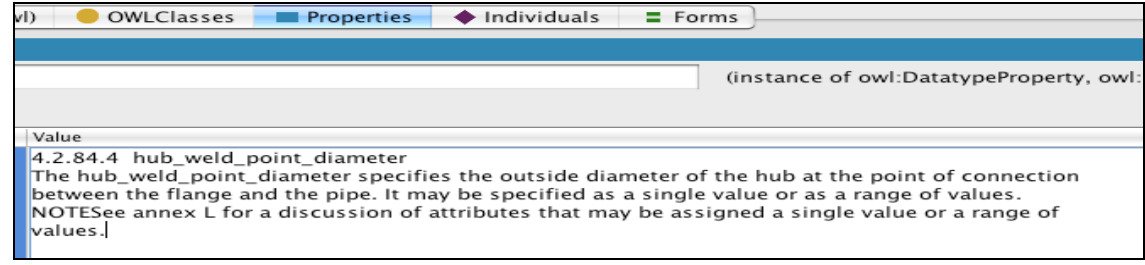

Fig. 4. Modeling of the Flange, data type properties

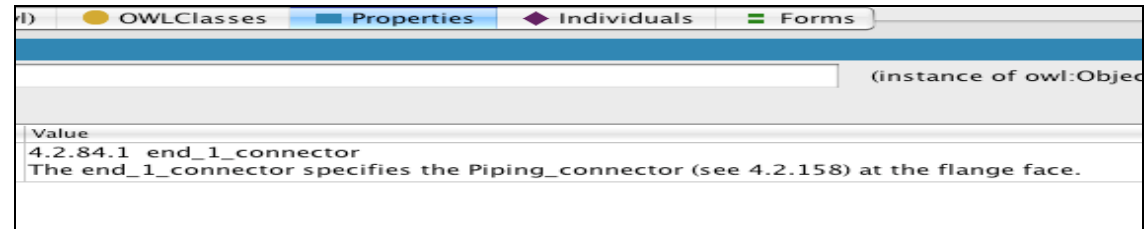

Fig. 5. Modeling of the Flange, relational properties

Stage 6- Knowledge Base transcription and refinement: For the example case, we decide than the Standard contains enough information for our modeling needs, hence no extension is needed. The process is finalized by running a reasoning process to check the ontology for any problems at a logical level (not shown here). Stage 7- Testing and instancing: In our case we use the Protégé OWL API for the generation of a java code suitable for the semi-automatic instancing of individuals, Fig. 6 (left) depict a piece of such code in the eclipse editor. Stage 8Application development based on Standards: as pointed before, this last stage comprises the actual usability of the Domain. In our case we used the modeled domain in order to match graphic elements coming from a 3D model with their parameters for a semantic synonym graphical adaptation as explained in [8]. Fig. 6 (Right), depicts such matching for the example. 

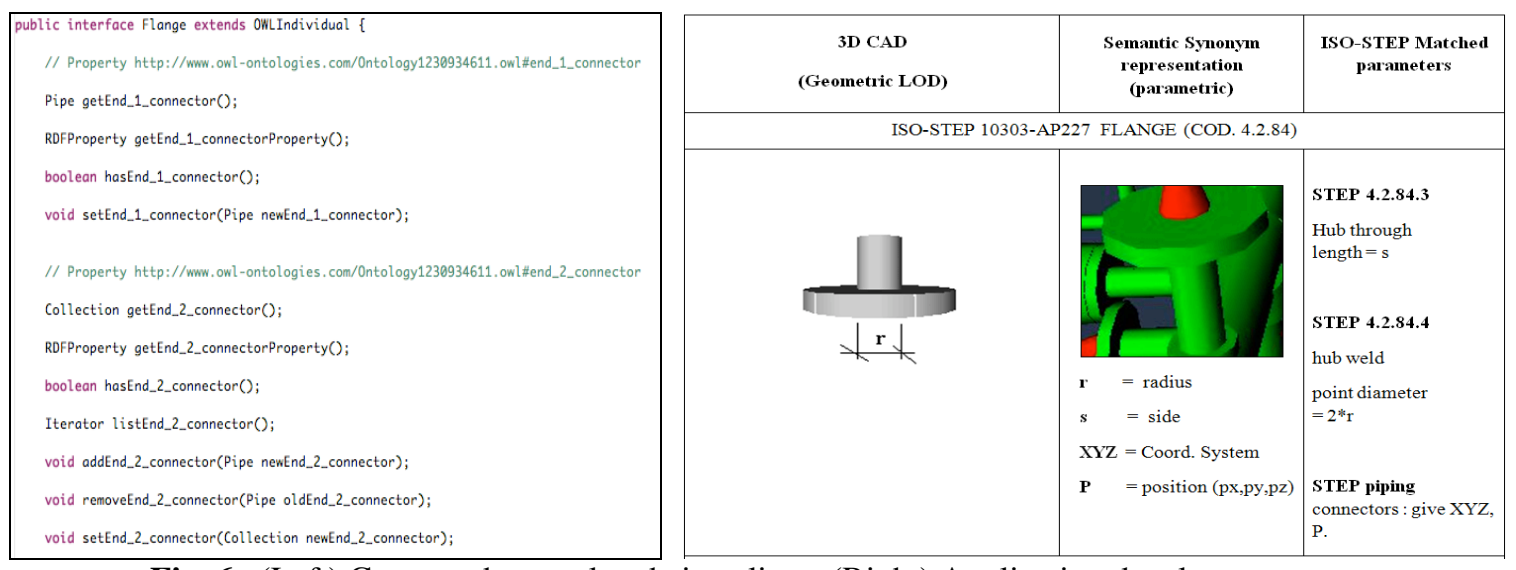

Fig. 6. (Left) Generated control code in eclipse, (Right) Application development

\section{Conclusions}

In this paper we presented a new methodology for the Domain modeling based on Engineering Standards. We discussed some benefits of standards as guidelines for a Knowledge Based Domain modeling and some potential challenges along with possible approaches to overcome them.

\section{References}

[1] BSI Standards, available online at: http://www.bsi-global.com/

[2] Feigenbaum, E. and McCorduck, P. The $5^{\circ}$ Generation Addison-Wesley, 1983

[3] Gruber, T., Toward Principles for the Design of Ontologies Used for Knowledge Sharing International Journal of Human-Computer Studies, 1995,

[4] Posada, J, Toro,C, Wundrak, S, and Stork, A. Ontology Supported Semantic Simplification of Large Data Sets of Industrial Plant CAD Models for Design Review Visualization. In KES 2005, volume 3681 Australia, 2005.

[5] Leroy, D. Standards and Publications for Engineering - Standards Organizations. Available online at: http://www.interfacebus.com/Standards

[6] Soanes-Stevenson (ed.), Oxford Dictionary of English, Oxford University Press, 2003

[7] Posada, J. A Methodology for the Semantic Visualization of Industrial Plant CAD Models for Virtual Reality Walkthroughs. Phd thesis, TU-Darmstadt, 2005.

[8] Toro, C, Posada, J, Oyarzun J, and Falcon, J. Supporting the CAD Structural Design Process with Knowledge-based Tools. Cybernetics and Systems, 38(5):575_586, 2007. 